\title{
Interaction of Tau with the chemokine receptor, CX3CR1 and its effect on microglial activation, migration and proliferation
}

\author{
Hariharakrishnan Chidambaram ${ }^{1,2}$, Rashmi Das ${ }^{1,2}$ and Subashchandrabose Chinnathambi ${ }^{1,2^{*}}$ (1)
}

\begin{abstract}
Alzheimer's disease (AD) is a neurodegenerative disease that leads to progressive loss of memory and dementia. The pathological hallmarks of AD include extracellular accumulation of amyloid- $\beta$ peptides forming senile plaques and intracellular accumulation of Tau oligomers and filamentous species. Tau is a microtubule-binding protein that stabilizes tubulin to form microtubules under physiological condition. In AD/ pathological condition, Tau detaches from microtubules and aggregates to form oligomers of different sizes and filamentous species such as paired helical filaments. Microglia are the resident brain macrophages that are involved in the phagocytosis of microbes, cellular debris, misfolded and aggregated proteins. Chemokine receptor, CX3CR1 is mostly expressed on microglia and is involved in maintaining the microglia in a quiescent state by binding to its ligand, fractalkine (CX3CL1), which is expressed in neurons as both soluble or membrane-bound state. Hence, under physiological conditions, the CX3CR1/CX3CL1 axis plays a significant role in maintaining the central nervous system (CNS) homeostasis. Further, CX3CR1/CX3CL1 signalling is involved in the synthesis of anti-inflammatory cytokines and also has a significant role in cytoskeletal rearrangement, migration, apoptosis and proliferation. In AD brain, the expression level of fractalkine is reduced, and hence Tau competes to interact with its receptor, CX3CR1. In microglia, phagocytosis and internalization of extracellular Tau species occurs in the presence of a chemokine receptor, CX3CR1 which binds directly to Tau and promotes its internalization. In this review, the pathophysiological roles of CX3CR1/fractalkine signalling in microglia and neurons at different stages of Alzheimer's disease and the possible role of CX3CR1/Tau signalling has been widely discussed.
\end{abstract}

Keywords: Alzheimer's disease, Microglia, Neuron, Tau, Fractalkine, CX3CR1 receptor

\section{Background}

Alzheimer's disease is a neurodegenerative disease that leads to progressive loss of memory and dementia [1]. Alzheimer's disease mostly occurs at the age of 65 and above, even though age is not the only factor for this disease [2]. Several other factors such as genetic and environmental factors (eg. stress) also play a critical role in the onset of Alzheimer's disease [3, 4]. Extracellular

\footnotetext{
*Correspondence: s.chinnathambi@ncl.res.in

${ }^{1}$ Neurobiology Group, Division of Biochemical Sciences, CSIR-National Chemical Laboratory, Dr. Homi Bhabha Road, 411008 Pune, India

Full list of author information is available at the end of the article
}

deposition of senile plaques from amyloid- $\beta(A \beta)$ peptides and intracellular accumulation of Tau oligomers and aggregates are the pathological features of Alzheimer's disease $[5,6]$. Amyloid precursor protein (APP) is a single transmembrane protein which is predominantly expressed in the central nervous system. Under physiological conditions, non-amyloidogenic APP processing occurs, which involves APP cleavage by $\alpha$ - and $\gamma$-secretases yielding soluble-APP $\alpha(\mathrm{sAPP} \alpha)$, APP intracellular domain (AICD) and p3 [7]. sAPP $\alpha$ secreted from cleavage of APP is involved in neuroprotection and neurite outgrowth $[8,9]$. In $\mathrm{AD}$, an imbalance occurs in the proteolysis of APP towards amyloidogenic processing 
that leads to the secretion of $A \beta$. The levels of $\alpha$-secretase had been reduced, which alters the proteolytic cleavage of APP by $\beta$ - and $\gamma$-secretases yielding $\operatorname{sAPP} \beta$, AICD and $A \beta[7,10]$. Proteolytic cleavage of APP by $\alpha$ - and $\beta$-secretases are regulated by several G-protein coupled receptors (GPCRs) that leads to secretion and accumulation of $A \beta$ plaques under pathological conditions [7]. Tau is a microtubule-binding protein that stabilizes tubulin to form microtubules in the neuronal cells $[11,12]$. In humans, Tau gene is located on chromosome 17q21. Tau is predominantly expressed in the axons of matured neurons and encodes for six different isoforms. Full-length Tau consists of 441 amino acids with an N-terminal domain, two N-terminal inserts (N1 and N2), two proline-rich domains (PRD1 and PRD2), four repeat regions that form microtubule-binding domain (R1-R4) and a $\mathrm{C}$-terminal domain. The isoforms are generated based on the alternative splicing of exons that encodes for N1, $\mathrm{N} 2$ inserts and $\mathrm{R} 2$ region of repeat domain to form $2 \mathrm{~N} 4 \mathrm{R}$ (hTau40), 1N4R (hTau 34), 0N4R (hTau 24), 2N3R (hTau 39), 1N3R (hTau 37) and 0N3R (hTau 23). Tau is a phosphoprotein that requires basal phosphorylation for its physiological functioning. In Alzheimer's disease, Tau undergoes several post-translational modifications such as hyperphosphorylation, glycation, glycosylation, nitration, methylation, sumoylation, truncation, etc., [13]. Among the various post-translational modifications, Tau phosphorylation is widely studied and majorly contributes to Tau pathology that leads to its detachment from microtubules, misfolding and intracellular accumulation to form oligomeric and filamentous species $[13,14]$. Currently, Tau-based therapy is attaining more attention over $A \beta$-based treatment and this led to the discovery of various mechanisms for inhibiting Tau-mediated pathology such as inhibition and reversal of post-translational modifications, aggregation inhibition and disaggregation of preformed fibrils [15-23].

\section{G-protein coupled receptors in Alzheimer's disease} GPCRs belong to the largest class of membrane proteins that are involved in cell-to-cell communications, signal transduction pathways and hormonal regulation [24, 25]. The human genome contains about 800 different GPCRs that are classified as five different classes (rhodopsin, secretin, glutamate, adhesion, and frizzled/taste receptors) [26]. GPCRs contain seven transmembrane domains made of $\alpha$-helices with an extracellular $\mathrm{N}$-terminal and intracellular C-terminal domain [27]. GPCRs respond to a variety of extracellular signals such as hormones, neurotransmitters, proteins, peptides, lipids, ions, photons, etc., by a change in structural conformation [28]. Upon GPCR activation, the bound G-proteins are activated by exchanging the GDP with a GTP and initiate the downstream signalling cascades such as activation/inhibition of secondary signalling molecules, cellular kinases, nuclear transcription factors, etc., [29] GPCR signalling is also mediated by $\beta$-arrestin, a G-protein independent pathway that involves phosphorylation of membrane GPCRs by GPCR kinases (GRKs), followed by binding of $\beta$-arrestin in the intracellular region [30]. $\beta$-arrestins are the scaffolding proteins that promote receptor desensitizing, internalization and activation of several other intracellular signals [31]. There are several other mechanisms of GPCR activation such as intracellular activation, transactivation, dimerization activation, biphasic activation and biased activation [32]. GPCRs are the major drug targets, and approximately 700 approved drugs targeting GPCRs accounts for 35\% of approved drug targets [33]. In AD, the pathophysiological role of GPCRs has been widely studied, and there are several reports stating that GPCRs are playing a significant role in both amyloid- $\beta$ and Tau hypothesis $[7,34,35]$. The cDNA microarray analysis obtained from $\mathrm{AD}$ patients also stated that the expression of several GPCRs had been altered when compared with controls [36]. Several neuronal GPCRs such as A1 adenosine receptor, Angiotensin II type-2 receptor, Metabotropic glutamate receptor-2, CXCR2 and CCR3 chemokine receptors are involved in Tau phosphorylation mediated by several cellular kinases such as GSK-3ß, ERKs and CDK5 kinases [34], whereas GPCRs such as M1 muscarinic acetylcholine receptor, $\beta 2$-adrenergic receptor and corticotropin releasing factor receptor-1 are involved in downregulation of Tau phosphorylation (briefly reviewed by Chidambaram et al. 2020) [34]. The neuronal GPCRs, muscarinic acetyl choline receptors were been hypothesized to interact with extracellular Tau resulting in the increased level of intracellular calcium on Tau exposure to neuroblastoma cells [37]. Later, identified that Tau activates M1 and M3 muscarinic acetylcholine receptors by its interaction and promotes intracellular calcium levels in neuronal cells. [37-39]. In addition, CX3CR1 chemokine receptor in microglia acts as surface receptor for extracellular Tau that interacts and promotes its internalization [40]. These evidences highlight the pathophysiological role of GPCRs in Tauopathy.

\section{Microglia-scavengers of misfolded proteins}

Microglia are the resident macrophages that act as innate immune cells of the brain and play a significant role in regulating brain development [41, 42]. Microglia are derived from primitive yolk sac progenitors and contributes to around $10-15 \%$ of total brain cells [43, 44]. Microglia are capable of changing the morphology in response to their microenvironment [45]. Microglia are primarily involved in constant surveillance of 
its microenvironment, phagocytosis of microbes, cellular debris, damaged cells and aggregated proteins [46]. Microglia are also involved in synaptic pruning, i.e. phagocytosis and clearance of excessive neuronal cells and synapse leading to proper neuronal function and synaptic plasticity in developing CNS [47]. In healthy condition, microglia are maintained at a quiescent stage by several intrinsic (Runt-related transcription factor-1, Interferon regulatory factor-8, etc.,) and extrinsic factors (CD200, CX3CR1, TREM2, etc.) [48]. Several cytokines and neurotransmitters released by neurons either as 'secreted' or 'membrane-bound' are involved in the neuron-glia cross talk to maintain microglial homeostasis $[49,50]$. Microglia are activated in response to a variety of factors present in their microenvironment. Microglial receptors such as toll-like receptors, Triggering receptor expressed on myeloid cells 2 (TREM-2), etc., receives the signals from the environment and activates microglia in response to infection or inflammation [51-53]. The cytokines from type-1 T-helper cells such as IFN- $\gamma$ and lipopolysaccharides are involved in proinflammatory activation of microglia (M1-microglia) and the cytokines from type-2 T-helper cells and dietary fatty acids are involved in the anti-inflammatory activation of microglia (M2-microglia) [44, 52-55]. In Alzheimer's disease, microglia have both beneficial as well as harmful effects depending on disease stage and progression. There are several reports stating that microglia are primarily involved in phagocytosis and clearance of plaques, fibrils and damaged cells [56, 57]. Microglial priming and presenting self-antigen such as Tau and amyloid- $\beta$ to the infiltrated CD4+ T lymphocyte cells through MHC I/II molecules alter its phenotype from M1 to form M2 stage which is involved in phagocytosis, tissue remodelling and neuroprotection [58]. Genome-wide association studies reported over 20 genetic loci that are related to $\mathrm{AD}$, among which several genes are selectively expressed in microglia $[59,60]$. TREM2 is one such receptor that plays a pivotal role in phagocytosis and clearance of apoptotic neurons, bacteria, lipoproteins, etc., [61-63]. Mutations in the TREM2 gene (R47H) increases the risk of AD that leads to impaired microglial activation and phagocytosis [64]. In microglia, there are various receptors involved in receptor-mediated endocytosis of amyloid- $\beta$. These include scavenger receptors, toll-like receptors, transmembrane receptors such as integrin and Triggering receptor expressed on myeloid cells 2 reported to interact directly with amyloid- $\beta$ fibrils for its activation, phagocytosis and internalization $[35,65,66]$. In $\mathrm{AD}$, extracellular Tau species activates microglia via actin remodelling, promotes migration and phagocytosis of Tau for its clearance which could further be enhanced by other factors such as lipids and fatty acids [67-69]. In Tauopathy models, microglia are involved in Tau propagation, neuroinflammation and neuronal damage by the release of toxic factors and pro-inflammatory cytokines and also leads to synaptic losses [70, 71]. Extracellular Tau treatment activates p38 MAPK and alters the pathway to express pro-inflammatory cytokines in primary microglia cells [72]. The gene expression of pro-inflammatory cytokines such as IL- 6 , IL- $1 \beta$, TNF- $\alpha$ and Mip- $1 \alpha$ has been increased in the microglial cells upon exposure to extracellular Tau [72]. Though there are not many studies performed on microglial surface receptors for Tau interaction and internalization, recent findings report that the chemokine receptor, CX3CR1 promotes Tau phagocytosis and internalization [40].

\section{CX3CR1 receptor-physiological functions in the CNS}

CX3CR1 is a chemokine receptor that belongs to the class A family of G-protein coupled receptors. In humans, CX3CR1 is abundantly expressed in white blood cells such as lymphocytes and monocytes for their migration [73]. In the CNS, CX3CR1 receptor is largely expressed by microglial cells (brain macrophages) followed by neuronal cells that are involved in cell adhesion and migration [74-77]. Fractalkine (CX3CL1), the ligand for this receptor is constitutively expressed by neuronal cells and to a lesser extent by astrocytes and are present as either membrane-bound or soluble form [78, 79]. Fractalkine consists of total 373 amino acids among which the $\mathrm{N}$-terminal domain and the mucin-like stalk forms the extracellular flanking residues. The $\mathrm{N}$-terminal domain constitutes 76 amino acid residues, mucin-like stalk constitutes 241 amino acid residues, the transmembrane region constitutes 19 amino acid residues, and the intracellular C-terminal region constitutes 37 amino acid residues [80]. The $\mathrm{N}$-terminal peptide and the mucinlike stalk of the membrane-bound protein are cleaved by many proteolytic enzymes such as cysteine proteases, cathepsin-S, and metalloproteinases such as disintegrin and metalloproteinase domain-containing protein 10 (ADAM10), disintegrin and metalloproteinase domaincontaining protein 17 (ADAM17) to form the soluble fractalkine [81-83]. The membrane-bound fractalkine acts as an adherent and maintains the microglia in resting state, whereas the soluble fractalkine acts as a chemoattractant promoting microglial migration towards the site of infection or inflammation [84]. This leads to the neuron-microglia cross talk in the development of brain such as learning, memory and synaptic plasticity [85]. CX3CR1 is a $G_{i}$ protein-bound GPCR that on fractalkine binding activates several signalling molecules and transcription factors such as nuclear factor kappa-lightchain-enhancer of activated $\mathrm{B}$ cells $(\mathrm{NFK} \mathrm{B})$ and cAMP 
response element-binding protein (CREB) $[85,86]$. The activated transcription factors are involved in regulating microglial activation, cytokine synthesis, migration, proliferation and neuronal survival. [77, 85, 86].

\section{CX3CR1/Fractalkine signalling-different roles in Microglia}

Fractalkine is involved in the inhibition of microglial activation through the CX3CR1 receptor by activating PI3K and Akt signals and maintains microglia in a quiescent state [87]. In aged rats, the level of fractalkine mRNA and fractalkine was reduced and observed with enhanced microglial activation, MHC II, CD40 mRNA levels and IL-1 $\beta$ secretion. Fractalkine treatment also reduced age-related increase in MHC II, CD40 mRNAs, IL-1 $\beta$ levels and reduced microglial activation in aged rats [87]. Fractalkine is reported to inhibit the release of TNF- $\alpha$, a pro-inflammatory cytokine released by LPS activated microglia [88]. Moreover, IL-1 $\beta$ (pro-inflammatory cytokine) synthesis by LPS activated microglia, and its neuronal receptor TLR-4 signalling is also reduced on fractalkine exposure in PI3K and Akt dependent manner that denotes the anti-inflammatory effect of CX3CL1 (both soluble and membrane-bound form) [87]. Under pathological conditions, TLR-4 signalling is reported to be involved in Tau phosphorylation through p38 MAP kinases that are regulated by CX3CL1 signalling [89]. In 2014, Becker et al. demonstrated that fractalkine treatment activates nuclear transcription factor such as nuclear factor erythroid 2-related factor 2 (NRF2) mediated by PI3K and Akt activation, GSK-3 $\beta$ inactivation in microglial cells [90]. These nuclear transcription factors are involved in the synthesis of antioxidant enzymes, including heme oxygenase 1 and attenuate the proinflammatory phenotype in microglia [90]. In 2018, controversial results were reported by the same group that CX3CR1 receptor signalling is involved in the synthesis of pro-inflammatory cytokines such as IL- $1 \beta$, TNF- $\alpha$, etc., mediated by MSK-1 and NFKB [91]. CX3CR1 signalling also triggered kinases such as ERK-1/2, p38 and JNK in immortal microglial (IMG) cells [91]. In addition, CX3CR1 deficient primary microglia had been observed with impaired NRF2, deficiency of phagocytosis and impaired microglial migration [92] (Fig. 1).

\section{CX3CR1/Fractalkine in neurons-role in neuroprotection, neurogenesis, learning, memory and synaptic plasticity}

Apart from the indirect effects of fractalkine on neuronal cells through microglial signalling, there are several direct roles of fractalkine in neuronal cells. In the CNS, fractalkine is involved in anti-inflammatory effects and protects neuronal cells against neuroinflammation and tissue damage. Fractalkine treatment to neuronal cells led to the activation of ERK and Akt kinases upon transient phosphorylation [93]. Fractalkine also has its role on other receptors such as NMDA receptors, Adenosine receptors, etc., It inhibits NMDA receptor-induced calcium influx and apoptosis; activates the transcription factors such as CREB and NFkB; ultimately involved in learning, memory and synaptic plasticity-mediated by ERK activation [93]. It promotes the release of adenosine from activated microglia, which in turn activates $\mathrm{A} 1$ adenosine receptor that is involved in neuroprotection [94]. Fractalkine is also involved in hippocampal neurogenesis that is involved in learning and memory. It also acts as neuroprotectant and protects the neuron from neuroinflammation and neuronal injury. Fractalkine expression in aged rats has been reduced, which led to increased CX3CR1 expression and microglial activation [95]. Similar conditions occur in advanced conditions of $\mathrm{AD}$, the expression of fractalkine reduces that increases microglial activation ultimately leading to neuronal inflammation, loss of synapses and cognitive deficits (Fig. 2).

\section{CX3CR1 knockout studies in AD models-role in A $\beta$ and Tau clearance}

Microglial CX3CR1/CX3CL1 axis plays a significant role in the progression of Alzheimer's disease with controversy in $A \beta$ and Tau pathology [96]. CX3CR1 impairment reduces $A \beta$-mediated pathology and promotes $A \beta$ clearance. In hTau/CX3CR1 $1^{-/-}$mice models, microglial activation by lipopolysaccharides promoted p38 MAPKmediated Tau hyperphosphorylation and behavioural impairments. Further, it is proved that microglial activation led to secretion of pro-inflammatory cytokine IL-1 that promotes Tau hyperphosphorylation and aggregation by TLR4-mediated pathway in neurons [89]. In hAPP mice models, CX3CR1 knockout promoted Tau pathology (hyperphosphorylation) and cognitive deficits with no significant changes in APP processing and levels of $A \beta$ [97], whereas Lee et al., demonstrated that CX3CR1 deficiency regulates $A \beta$ deposition, promotes microglial phagocytosis and A $\beta$ clearance in APPPS-1 and R1.40 mice models [98]. Further, A $\beta$ significantly reduced CX3CR1 expression and impaired CX3CR1 signalling in cultured microglial cells and AD brain [97].

\section{CX3CR1/Tau signalling-role in pro-inflammatory cytokine synthesis, microglial activation and migration}

In $\mathrm{AD}$ brain, neurons undergo severe inflammation and tissue damage that spreads aggregated Tau species such as oligomers and filaments around the microglial environment. Bolos et al. demonstrated that extracellular Tau is capable of binding to $\mathrm{N}$-terminal peptides 

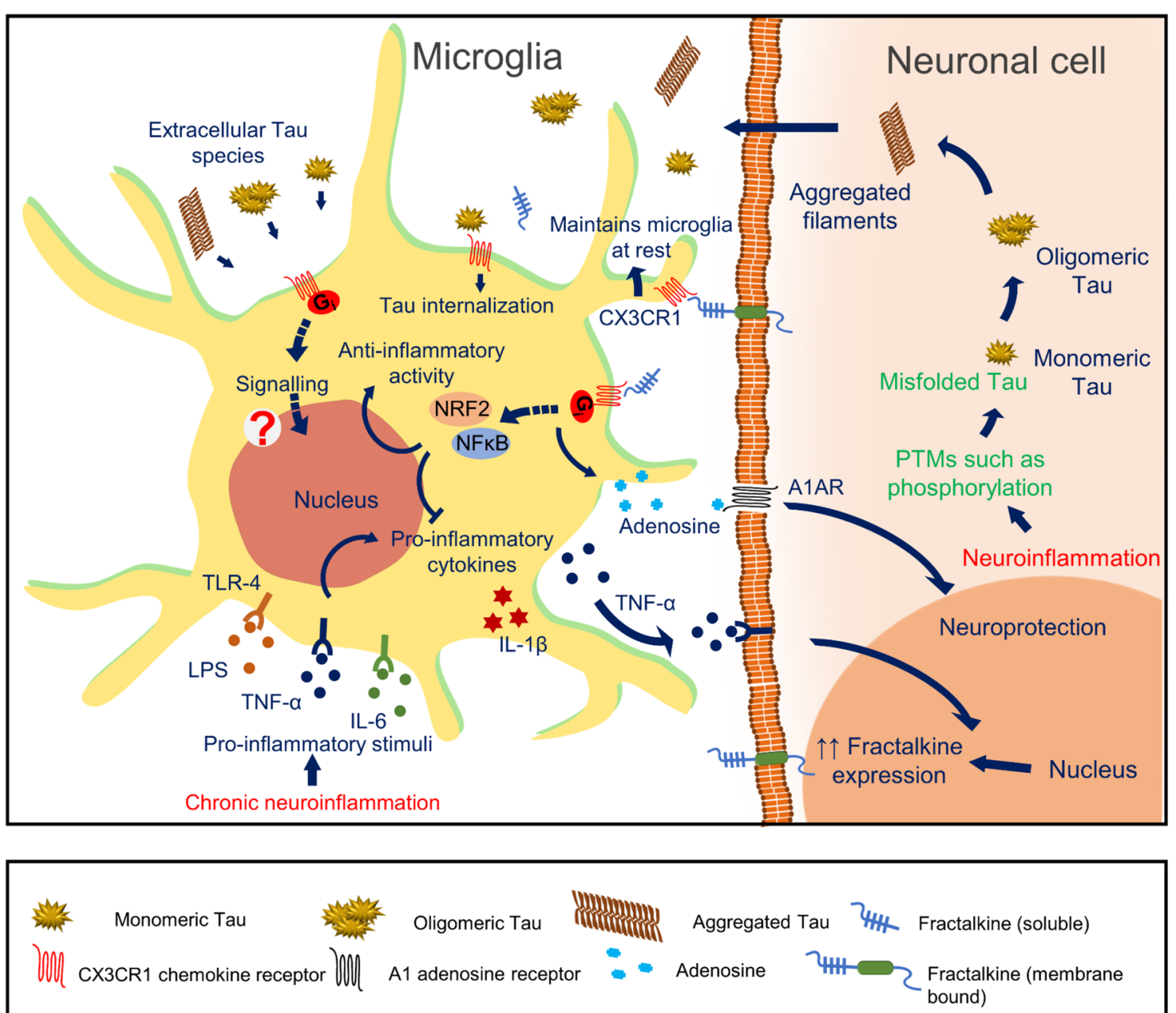

Fig. 1 CX3CR1/ Fractalkine signalling — different roles in Microglia: In Alzheimer's disease, chronic neuroinflammation increases the pro-inflammatory stimuli such as LPS, TNF- $\alpha$ and IL-6 in the CNS. Microglial cells, which are maintained at the resting stage under normal conditions, are activated by these pro-inflammatory stimuli by interacting through several surface receptors. Upon pro-inflammatory activation, microglia release excess of pro-inflammatory cytokines in the environment to activate more microglial cells to target the site of inflammation. Pro-inflammatory cytokine, IL-1 $\beta$ secreted from inflammatory microglia activates the TLR4 signalling pathway of neuronal cells and is involved in hyperphosphorylation of Tau protein by activating p38 MAP kinases. Fractalkine (CX3CL1), constitutively expressed neuronal protein, is either present as soluble or membrane-bound maintains the microglia in a quiescent stage. The pro-inflammatory TNF-a secretion by the microglial cells activates TNF receptors of neuronal cells and promotes the expression of fractalkine. Fractalkine interacts with CX3CR1 and activates $\mathrm{G}_{i}$ protein-mediated signalling pathway that activates nuclear transcription factors, NFKB and NRF2. CX3CR1/CX3CL1 activation also inhibits pro-inflammatory cytokine synthesis and microglial activation. In AD conditions, misfolded Tau aggregated to form oligomers and filaments that spread across the CNS. Also, the extracellular Tau interacts with CX3CR1 for its internalization

of CX3CR1 and promotes its internalization in microglial cells [40]. In AD brain, the expression level of fractalkine is reduced, and hence Tau competes to interact with this receptor. Further, CX3CR1/CX3CL1 signalling is involved in the synthesis of anti-inflammatory cytokines and also has a significant role in cytoskeletal rearrangement, migration, apoptosis and proliferation. On Tau exposure to microglial cells, the p38 pathway gets altered and activated with a significant change in the pro-inflammatory cytokine expression profile [72]. Pro-inflammatory cytokines such as Il-6, IL-1 $\beta$, TNF- $\alpha$ and Mip- $1 \alpha$ has been synthesized by these microglial cells [72]. Similarly, in AD, highly accumulated extracellular Tau interacts with the chemokine receptor, CX3CR1 and prevents CX3CL1 from binding to its receptor. We hypothesize that CX3CR1/Tau signalling could be involved in the synthesis of pro-inflammatory cytokines, enhanced microglial activation and 

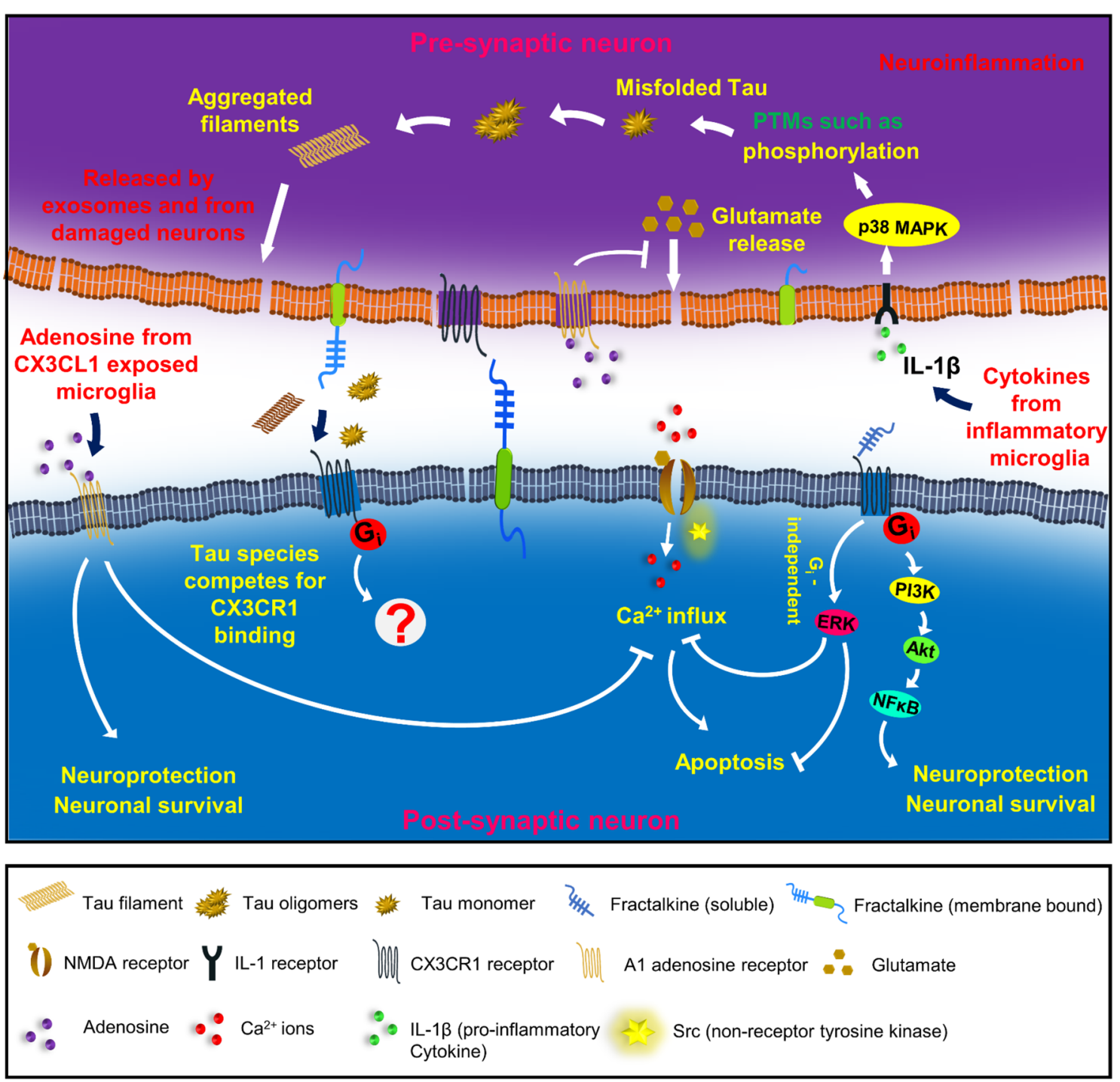

Fig. 2 CX3CR1/ Fractalkine signalling — pathophysiological roles in neurons: In neuronal cells, fractalkine plays a significant role in neuroprotection, neurogenesis, learning, memory and synaptic plasticity. The chemokine receptor is involved in $\mathrm{G}_{i}$-protein-mediated signalling that leads to the activation of the transcription factor, NFkB mediated by PI3K and Akt signals. NFKB is involved in neuronal survival and neuroprotective effects. Fractalkine is also involved in Gi-protein independent activation of ERK kinases that inhibits NMDA receptor-mediated calcium influx and neuronal apoptosis. Fractalkine exposed microglia releases adenosine that is involved in neurotrophic effects via A1 adenosine signalling mechanisms. In neurodegenerative diseases, neurons undergo chronic inflammation, and Tau forms oligomers and filaments due to various post-translational modifications such as phosphorylation. Inflammatory microglia release several pro-inflammatory cytokines such as IL-1 $\beta$, TNF-a and IL-6. IL-1 $\beta$ promotes Tau phosphorylation and aggregation mediated by IL-1 and TLR-4 receptors. Extracellular Tau interacts with CX3CR1 receptor in microglia for its internalization. We hypothesize that extracellular Tau species may also have possible interaction with neuronal receptor and differential signalling towards neuronal inflammation and neurodegeneration

migration that ultimately leads to neuro-inflammation and neuronal damage in neurodegenerative diseases such as Alzheimer's disease.

\section{Conclusion}

Microglia plays a critical role in the progression of Alzheimer's disease. Microglia are capable of internalizing and degrading both $\mathrm{A} \beta$ deposits and Tau aggregates [14, $35,40]$. In $\mathrm{AD}$, neuroinflammation leads to excess activation of microglia promoting migration, cell proliferation and impaired phagocytosis. The role of extracellular 
Tau species in CX3CR1 receptor interaction, signalling, inflammatory activation and proliferation of microglia in Alzheimer's disease is still poorly understood and further needs to be addressed. This study would help to know the mechanism of CX3CR1/Tau signalling in impaired phagocytosis, enhanced migration, proliferation and neuroinflammation.

\begin{abstract}
Abbreviations
AD: Alzheimer's disease; AB: Amyloid- $\beta$; CX3CL1: Fractalkine; CNS: Central nervous system; APP: Amyloid precursor protein; sAPPa: Soluble amyloid precursor protein-a; AICD: APP intracellular domain; GPCRs: G-protein coupled receptors; PRD: Proline-rich domain; GRKs: G-protein coupled receptor kinases; GSK-3B: Glycogen synthase kinase-3 $\beta$; ERKs: Extracellular signal-regulated kinases; CDK5: Cyclin-dependent kinase-5; ADAM10: A disintegrin and metalloproteinase domain-containing protein 10; ADAM17: A disintegrin and metalloproteinase domain-containing protein 17; MSK-1: Mitogen- and stress-activated kinase-1; TLR-4: Toll-like receptor-4; TREM-2: Triggering receptor expressed on myeloid cells 2; NRF-2: Nuclear factor erythroid 2 (NFE2)-related factor 2; CREB: CAMP responsive element binding protein; NFKB: Nuclear factor kappalight-chain-enhancer of activated B cells; PI3K: Phosphoinositol-3 kinase; Akt:

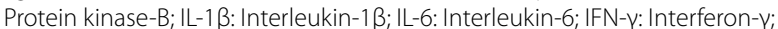
TNF-a: Tumour necrosis factor-a; Mip-1 a: Macrophage inflammatory protein 1-alpha; CD200: Cluster of differentiation 200; CD40: Cluster of differentiation 40; MHC: Major histocompatibility complex.
\end{abstract}

\section{Acknowledgements}

We are grateful to Chinnathambi's lab members for their scientific discussions, helpful suggestions and critical reading of the manuscript.

\section{Authors' contributions}

$\mathrm{HC}$ and $\mathrm{SC}$ performed the literature search and wrote the manuscript. RD helped in the discussion part. SC conceived the idea of the work and supervised the project. We are grateful to Chinnathambi's lab members for their critical readings and fruitful discussions. All authors read and approved the final manuscript.

\section{Funding}

This project is supported in part by grant from in-house CSIR-National Chemical Laboratory grant MLP029526. Hariharakrishnan Chidambaram acknowledges the fellowship from Department of Biotechnology, Government of India.

\section{Availability of data and materials \\ Not applicable.}

Ethics approval and consent to participate

Not applicable.

\section{Consent for publication}

Not applicable.

\section{Competing interests}

The authors declare that they have no competing interests.

\section{Author details \\ ${ }^{1}$ Neurobiology Group, Division of Biochemical Sciences, CSIR-National Chemical Laboratory, Dr. Homi Bhabha Road, 411008 Pune, India. ${ }^{2}$ Academy} of Scientific and Innovative Research (AcSIR), 411008 Pune, India.

Received: 25 August 2020 Accepted: 8 September 2020

Published online: 15 September 2020

\section{References}

1. Hampel H, Schneider LS, Giacobini E, Kivipelto M, Sindi S, Dubois B, et al. Advances in the therapy of Alzheimer's disease: targeting amyloid beta and tau and perspectives for the future. Expert Rev Neurother. 2015;15(1):83-105.

2. Sloane PD, Zimmerman S, Suchindran C, Reed P, Wang L, Boustani M, et al. The public health impact of Alzheimer's disease, 2000-2050: potential implication of treatment advances. Ann Rev Public Health. 2002;23:213.

3. Bertram L, Lill CM, Tanzi RE. The genetics of Alzheimer disease: back to the future. Neuron. 2010;68(2):270-81.

4. Scaccianoce S, Caruso A, Nicoletti F, Gaetano A. Risk factors for Alzheimer's disease: focus on stress. Front Pharmacol. 2019;10:976.

5. Serrano-Pozo A, Frosch MP, Masliah E, Hyman BT. Neuropathological alterations in Alzheimer disease. Cold Spring Harbor Perspect Med. 2011;1(1):a006189.

6. Skender-Gazibara M, Slobodan D. Neuropathological hallmarks of Alzheimer's disease. Arch Oncol. 2001;9(1):5-7.

7. Thathiah A, De Strooper B. The role of G protein-coupled receptors in the pathology of Alzheimer's disease. Nat Rev Neurosci. 2011;12(2):73-877.

8. Furukawa K, Sopher BL, Rydel RE, Begley JG, Pham DG, Martin GM, et al. Increased activity-regulating and neuroprotective efficacy of a-secretasederived secreted amyloid precursor protein conferred by a C-terminal heparin-binding domain. J Neurochem. 1996;67(5):1882-966.

9. Small DH, Nurcombe V, Reed G, Clarris H, Moir R, Beyreuther K, et al. A heparin-binding domain in the amyloid protein precursor of Alzheimer's disease is involved in the regulation of neurite outgrowth. J Neurosci. 1994;14(4):2117-27.

10. Sennvik K, Fastbom J, Blomberg M, Wahlund L-O, Winblad B, Benedikz E. Levels of $\alpha$-and $\beta$-secretase cleaved amyloid precursor protein in the cerebrospinal fluid of Alzheimer's disease patients. Neurosci Lett. 2000;278(3):169-72.

11. Binder LI, Frankfurter A, Rebhun LI. The distribution of tau in the mammalian central nervous system. J Cell Biol. 1985;101(4):1371-8.

12. Conde C, Cáceres A. Microtubule assembly, organization and dynamics in axons and dendrites. Nat Rev Neurosci. 2009;10(5):319-32.

13. Martin L, Latypova X, Terro F. Post-translational modifications of tau protein: implications for Alzheimer's disease. Neurochem Int. 2011;58(4):458-71.

14. Mroczko B, Groblewska M, Litman-Zawadzka A. The role of protein misfolding and Tau Oligomers (TauOs) in Alzheimer's disease (AD). Int J Mol Sci. 2019;20(19):4661.

15. Balmik AA, Das R, Dangi A, Gorantla NV, Marelli UK, Chinnathambi S. Melatonin interacts with repeat domain of Tau to mediate disaggregation of paired helical filaments. Biochem Biophys Acta. 2020;1864(3):129467.

16. Brunden KR, Trojanowski JQ, Lee VMY. Advances in tau-focused drug discovery for Alzheimer's disease and related tauopathies. Nat Rev Drug Discov. 2009;8(10):783-93.

17. Congdon EE, Sigurdsson EM. Tau-targeting therapies for Alzheimer disease. Nat Rev Neurol. 2018;14(7):399-415.

18. Gorantla NV, Das R, Chidambaram H, Dubey T, Mulani FA, Thulasiram HV, et al. Basic limonoid modulates chaperone-mediated proteostasis and dissolve Tau fibrils. Sci Rep. 2020:10(1):1-19.

19. Gorantla NV, Landge VG, Nagaraju PG, Priyadarshini CGP, Balaraman E, Chinnathambi S. Molecular cobalt (II) complexes for tau polymerization in Alzheimer's disease. ACS Omega. 2019;4(16):16702-14.

20. Noble W, Jimenez-Sanchez M, Perez-Nievas BG, Hanger DP. Considerations for future tau-targeted therapeutics: can they deliver?. Abingdon: Taylor \& Francis; 2020.

21. Panza F, Solfrizzi V, Seripa D, Imbimbo BP, Lozupone M, Santamato A, et al. Tau-centric targets and drugs in clinical development for the treatment of Alzheimer's disease. BioMed Res Int. 2016;2016:3245935.

22. Sonawane SK, Balmik AA, Boral D, Ramasamy S, Chinnathambi S. Baicalein suppresses Repeat Tau fibrillization by sequestering oligomers. Arch Biochem Biophys. 2019;675:108119.

23. Das R, Balmik AA, Chinnathambi S. Melatonin reduces GSK3 $\beta$ expression and tau phosphorylation via Nrf2 nuclear translocation. bioRxiv. 2019;861229.

24. Ji TH, Grossmann M, Ji I. G protein-coupled receptors I. Diversity of receptor-ligand interactions. J Biol Chem. 1998;273(28):17299-302. 
25. Tse LH, Wong YH. GPCRs in Autocrine and Paracrine Regulations. Front Endocrinol. 2019;10:428.

26. Fredriksson R, Lagerström MC, Lundin L-G, Schiöth HB. The G-proteincoupled receptors in the human genome form five main families. Phylogenetic analysis, paralogon groups, and fingerprints. Mol Pharmacol. 2003;63(6):1256-72.

27. Kobilka BK. G protein coupled receptor structure and activation. Biochem Biophys Acta. 2007;1768(4):794-807.

28. Civelli O, Reinscheid RK, Zhang Y, Wang Z, Fredriksson R, Schiöth HB. $G$ protein-coupled receptor deorphanizations. Annu Rev Pharmacol Toxicol. 2013;53:127-46.

29. Lambright DG, Noel JP, Hamm HE, Sigler PB. Structural determinants for activation of the a-subunit of a heterotrimeric $\mathrm{G}$ protein. Nature. 1994;369(6482):621.

30. Dorn GW. GRK mythology: G-protein receptor kinases in cardiovascular disease. J Mol Med. 2009;87(5):455-63.

31. DeWire SM, Ahn S, Lefkowitz RJ, Shenoy SK. Beta-arrestins and cell signaling. Annu Rev Physiol. 2007;69:483.

32. Wang W, Qiao Y, Li Z. New insights into modes of GPCR activation. Trends Pharmacol Sci. 2018;39(4):367-86.

33. Sriram K, Insel PA. G protein-coupled receptors as targets for approved drugs: how many targets and how many drugs? Mol Pharmacol. 2018;93(4):251-8.

34. Chidambaram H, Chinnathambi S. G-Protein coupled receptors and Taudifferent roles in Alzheimer's disease. Neuroscience. 2020;438:198.

35. Zhao $Y, W u X, L i X$, Jiang L-L, Gui $X$, Liu $Y$, et al. TREM2 is a receptor for $\beta$-amyloid that mediates microglial function. Neuron. 2018;97(5):1023-31.

36. Blalock E, Geddes J, Chen K, Porter N. Markesbery WR andLandfield PW Incipient Alzheimer's disease: Microarray correlation analyses reveal major transcriptional and tumor suppressor responses. Proc Natl Acad Sci. 2004:101:2173-8.

37. Gómez-Ramos A, Díaz-Hernández M, Cuadros R, Hernández F, Avila J. Extracellular tau is toxic to neuronal cells. FEBS Lett. 2006;580(20):4842-50.

38. Gómez-Ramos A, Díaz-Hernández M, Rubio A, Díaz-Hernández JI, Miras-Portugal MT, Avila J. Characteristics and consequences of muscarinic receptor activation by tau protein. Eur Neuropsychopharmacol. 2009:19(10):708-17.

39. Gomez-Ramos A, Diaz-Hernandez M, Rubio A, Miras-Portugal M, Avila J. Extracellular tau promotes intracellular calcium increase through M1 and M3 muscarinic receptors in neuronal cells. Mol Cell Neurosci. 2008:37(4):673-81.

40. Bolós M, Llorens-Martín M, Perea JR, Jurado-Arjona J, Rábano A, Hernández F, et al. Absence of CX3CR1 impairs the internalization of Tau by microglia. Mol Neurodegener. 2017;12(1):59.

41. Kofler J, Wiley CA. Microglia: key innate immune cells of the brain. Toxicol Pathol. 2011:39(1):103-14.

42. Lenz KM, Nelson LH. Microglia and beyond: innate immune cells as regulators of brain development and behavioral function. Front Immunol. 2018;9:698.

43. Alliot F, Godin I, Pessac B. Microglia derive from progenitors, originating from the yolk sac, and which proliferate in the brain. Dev Brain Res. 1999;117(2):145-52

44. Subramaniam SR, Federoff HJ. Targeting microglial activation states as a therapeutic avenue in Parkinson's disease. Front Aging Neurosci. 2017;9:176.

45. Town T, Nikolic $V$, Tan J. The microglial" activation" continuum: from innate to adaptive responses. J Neuroinflammation. 2005;2(1):24.

46. Arcuri C, Mecca C, Bianchi R, Giambanco I, Donato R. The pathophysiological role of microglia in dynamic surveillance, phagocytosis and structural remodeling of the developing CNS. Front Mol Neurosci. 2017;10:191.

47. Hong S, Dissing-Olesen L, Stevens B. New insights on the role of microglia in synaptic pruning in health and disease. Curr Opin Neurobiol. 2016:36:128-34.

48. Kierdorf K, Prinz M. Factors regulating microglia activation. Front Cell Neurosci. 2013;7:44

49. Pocock JM, Kettenmann H. Neurotransmitter receptors on microglia. Trends Neurosci. 2007:30(10):527-35

50. Sunnemark D, Eltayeb S, Nilsson M, Wallström E, Lassmann $\mathrm{H}$, Olsson T, et al. CX 3 CL1 (fractalkine) and CX 3 CR1 expression in myelin oligodendrocyte glycoprotein-induced experimental autoimmune encephalomyelitis: kinetics and cellular origin. J Neuroinflammation. 2005;2(1):17.

51. Fu R, Shen Q, Xu P, Luo JJ, Tang Y. Phagocytosis of microglia in the central nervous system diseases. Mol Neurobiol. 2014;49(3):1422-34.

52. Mosser DM, Edwards JP. Exploring the full spectrum of macrophage activation. Nat Rev Immunol. 2008;8(12):958.

53. Nakagawa $\mathrm{Y}$, Chiba K. Role of microglial $\mathrm{m} 1 / \mathrm{m} 2$ polarization in relapse and remission of psychiatric disorders and diseases. Pharmaceuticals. 2014:7(12):1028-48.

54. Gordon S, Martinez FO. Alternative activation of macrophages: mechanism and functions. Immunity. 2010;32(5):593-604.

55. Desale SE, Chinnathambi S. Role of dietary fatty acids in microglial polarization in Alzheimer's disease. J Neuroinflammation. 2020;17(1):1-14.

56. Colonna M, Butovsky O. Microglia function in the central nervous system during health and neurodegeneration. Annu Rev Immunol. 2017:35:441-68.

57. Nayak D, Roth TL, McGavern DB. Microglia development and function. Annu Rev Immunol. 2014:32:367.

58. Das R, Chinnathambi S. Microglial priming of antigen presentation and adaptive stimulation in Alzheimer's disease. Cell Mol Life Sci. 2019;76:3681-94

59. Hansen DV, Hanson JE, Sheng M. Microglia in Alzheimer's disease. J Cell Biol. 2018;217(2):459-72.

60. Lambert J-C, Ibrahim-Verbaas CA, Harold D, Naj AC, Sims R, Bellenguez C, et al. Meta-analysis of 74,046 individuals identifies 11 new susceptibility loci for Alzheimer's disease. Nat Genet. 2013;45(12):1452-8.

61. Kleinberger G, Yamanishi Y, Suárez-Calvet M, Czirr E, Lohmann E, Cuyvers E, et al. TREM2 mutations implicated in neurodegeneration impair cell surface transport and phagocytosis. Sci TransI Med. 2014;6(243):243ra86.

62. N'Diaye E-N, Branda CS, Branda SS, Nevarez L, Colonna M, Lowell C, et al. TREM-2 (triggering receptor expressed on myeloid cells 2 ) is a phagocytic receptor for bacteria. J Cell Biol. 2009;184(2):215-23.

63. Takahashi K, Rochford CD, Neumann H. Clearance of apoptotic neurons without inflammation by microglial triggering receptor expressed on myeloid cells-2. J Exp Med. 2005;201(4):647-57.

64. Jonsson T, Stefansson H, Steinberg S, Jonsdottir I, Jonsson PV, Snaedal J, et al. Variant of TREM2 associated with the risk of Alzheimer's disease. N Engl J Med. 2013;368(2):107-16

65. Bamberger ME, Harris ME, MCDonald DR, Husemann J, Landreth GE. A cell surface receptor complex for fibrillar $\beta$-amyloid mediates microglial activation. J Neurosci. 2003;23(7):2665-744.

66. Ries M, Sastre M. Mechanisms of A $\beta$ clearance and degradation by glial cells. Front Aging Neurosci. 2016;8:160.

67. Das R, Balmik AA, Chinnathambi S. Phagocytosis of full-length Tau oligomers by Actin-remodeling of activated microglia. J Neuroinflammation. 2020;17(1):1-15.

68. Desale SE, Chinnathambi S. a-Linolenic acid modulates phagocytosis of extracellular tau and induces microglial migration. bioRxiv.2020;042143.

69. Desale SE, Chinnathambi S. Dietary fatty acids mediate the secondary messenger phosphatidylinositol for microglial phagocytosis and migration. 2020;2020070532.

70. Leyns CE, Holtzman DM. Glial contributions to neurodegeneration in tauopathies. Mol Neurodegener. 2017:12(1):1-16.

71. Spangenberg EE, Green KN. Inflammation in Alzheimer's disease: lessons learned from microglia-depletion models. Brain Behav Immun. 2017:61:1-11.

72. Perea JR, Ávila J, Bolós M. Dephosphorylated rather than hyperphosphorylated Tau triggers a pro-inflammatory profile in microglia through the p38 MAPK pathway. Exp Neurol. 2018;310:14-211.

73. Bjarnadóttir TK, Gloriam DE, Hellstrand $\mathrm{SH}$, Kristiansson $\mathrm{H}$, Fredriksson $\mathrm{R}$, Schiöth HB. Comprehensive repertoire and phylogenetic analysis of the $\mathrm{G}$ protein-coupled receptors in human and mouse. Genomics. 2006;88(3):263-73.

74. Imai T, Hieshima K, Haskell C, Baba M, Nagira M, Nishimura M, et al. Identification and molecular characterization of fractalkine receptor CX3CR1, which mediates both leukocyte migration and adhesion. Cell. 1997:91(4):521-30.

75. Ostuni MA, Guellec J, Hermand P, Durand P, Combadière $C$, Pincet $F$, et al. CX3CL1, a chemokine finely tuned to adhesion: critical roles of the stalk glycosylation and the membrane domain. Biol Open. 2014;3(12):1173-82. 
76. Ransohoff RM. Chemokines and chemokine receptors: standing at the crossroads of immunobiology and neurobiology. Immunity. 2009:31(5):711-21.

77. Meucci O, Fatatis A, Simen AA, Miller RJ. Expression of CX3CR1 chemokine receptors on neurons and their role in neuronal survival. Proc Natl Acad Sci. 2000;97(14):8075-80.

78. Harrison JK, Jiang Y, Chen S, Xia Y, Maciejewski D, McNamara RK, et al. Role for neuronally derived fractalkine in mediating interactions between neurons and CX3CR1-expressing microglia. Proc Natl Acad Sci. 1998;95(18):10896-901.

79. Hatori K, Nagai A, Heisel R, Ryu JK, Kim SU. Fractalkine and fractalkine receptors in human neurons and glial cells. J Neurosci Res. 2002:69(3):418-26.

80. Umehara H, Bloom ET, Okazaki T, Nagano Y, Yoshie O, Imai T. Fractalkine in vascular biology: from basic research to clinical disease. Arterioscler Thromb Vasc Biol. 2004;24(1):34-40.

81. Clark AK, Yip PK, Malcangio M. The liberation of fractalkine in the dorsal horn requires microglial cathepsin S. J Neurosci. 2009;29(21):6945-54.

82. Garton K. TACE (ADAM17) mediates the cleavage and shedding of Fractalkine (CX3CL1). J Biol Chem. 2001:276:37993.

83. Hundhausen C, Misztela D, Berkhout TA, Broadway N, Saftig P, Reiss K, et al. The disintegrin-like metalloproteinase ADAM10 is involved in constitutive cleavage of CX3CL1 (fractalkine) and regulates CX3CL1-mediated cell-cell adhesion. Blood. 2003;102(4):1186-95.

84. Hermand $P$, Pincet $F$, Carvalho S, Ansanay $H$, Trinquet $E$, Daoudi M, et al. Functional adhesiveness of the $\mathrm{CX} 3 \mathrm{CL} 1$ chemokine requires its aggregation role of the transmembrane domain. J Biol Chem. 2008;283(44):30225-34.

85. Sheridan GK, Murphy KJ. Neuron-glia crosstalk in health and disease: fractalkine and CX3CR1 take centre stage. Open Biol. 2013;3(12):130181.

86. Poniatowski ŁA, Wojdasiewicz P, Krawczyk M, Szukiewicz D, Gasik R, Kubaszewski $Ł$, et al. Analysis of the role of CX3CL1 (Fractalkine) and its receptor CX3CR1 in traumatic brain and spinal cord injury: Insight into recent advances in actions of neurochemokine agents. Mol Neurobiol. 2017:54(3):2167-88.

87. Lyons A, Lynch AM, Downer EJ, Hanley R, O'Sullivan JB, Smith A, et al. Fractalkine-induced activation of the phosphatidylinositol-3 kinase pathway attentuates microglial activation in vivo and in vitro. J Neurochem. 2009;110(5):1547-56.

88. Zujovic V, Benavides J, Vigé X, Carter C, Taupin V. Fractalkine modulates TNF-a secretion and neurotoxicity induced by microglial activation. Glia. 2000;29(4):305-15.
89. Bhaskar K, Konerth M, Kokiko-Cochran ON, Cardona A, Ransohoff RM, Lamb BT. Regulation of tau pathology by the microglial fractalkine receptor. Neuron. 2010;68(1):19-311.

90. Lastres-Becker I, Innamorato NG, Jaworski T, Rabano A, Kügler S, Van Leuven F, et al. Fractalkine activates NRF2/NFE2L2 and heme oxygenase 1 to restrain tauopathy-induced microgliosis. Brain. 2014;137(1):78-91.

91. Castro-Sánchez S, García-Yagüe ÁJ, Kügler S, Lastres-Becker I. Impaired Signaling of NF-KB and NRF2 in CX3CR1-Deficient Microglia: implications in Tauopathies. bioRxiv. 2018;346304.

92. Castro-Sánchez S, García-Yagüe ÁJ, Kügler S, Lastres-Becker I. CX3CR1deficient microglia shows impaired signalling of the transcription factor NRF2: Implications in tauopathies. Redox Biol. 2019;22:101118.

93. Deiva K, Geeraerts T, Salim H, Leclerc P, Héry C, Hugel B, et al. Fractalkine reduces $\mathrm{N}$-methyl-d-aspartate-induced calcium flux and apoptosis in human neurons through extracellular signal-regulated kinase activation. Eur J Neurosci. 2004;20(12):3222-32.

94. Lauro C, Di Angelantonio S, Cipriani R, Sobrero F, Antonilli L, Brusadin V, et al. Activity of adenosine receptors type 1 is required for CX3CL1-mediated neuroprotection and neuromodulation in hippocampal neurons. J Immunol. 2008;180(11):7590-6.

95. Bachstetter AD, Morganti JM, Jernberg J, Schlunk A, Mitchell SH, Brewster $\mathrm{KW}$, et al. Fractalkine and CX3CR1 regulate hippocampal neurogenesis in adult and aged rats. Neurobiol Aging. 2011;32(11):2030-44.

96. Szepesi Z, Manouchehrian O, Bachiller S, Deierborg T. Bidirectional microglia-neuron communication in health and disease. Front Cell Neurosci. 2018;12:323.

97. Cho S-H, Sun B, Zhou Y, Kauppinen TM, Halabisky B, Wes P, et al. CX3CR1 protein signaling modulates microglial activation and protects against plaque-independent cognitive deficits in a mouse model of Alzheimer disease. J Biol Chem. 2011;286(37):32713-22.

98. Lee S, Varvel NH, Konerth ME, Xu G, Cardona AE, Ransohoff RM, et al. CX3CR1 deficiency alters microglial activation and reduces beta-amyloid deposition in two Alzheimer's disease mouse models. Am J Pathol. 2010;177(5):2549-62

\section{Publisher's Note}

Springer Nature remains neutral with regard to jurisdictional claims in published maps and institutional affiliations.
Ready to submit your research? Choose BMC and benefit from:

- fast, convenient online submission

- thorough peer review by experienced researchers in your field

- rapid publication on acceptance

- support for research data, including large and complex data types

- gold Open Access which fosters wider collaboration and increased citations

- maximum visibility for your research: over $100 \mathrm{M}$ website views per year

At BMC, research is always in progress.

Learn more biomedcentral.com/submissions 